\title{
Reconfigurable plasma antennas
}

\author{
Mohammad pourbagher $^{1}$, Javad nourinia ${ }^{1}$ and Nader pourmahmud ${ }^{2}$ \\ ${ }^{1}$ Department of Electrical Engineering, Urima University, Iran \\ ${ }^{2}$ Department of Mechanical Engineering, Urima University, Iran \\ armanpurbaqer@yahoo.com, j.nourinia@urmia.ac.ir,n.pormahmod@urmia.ac.ir
}

\begin{abstract}
This paper reviews recent work and applications of plasma antennas. Plasma antennas use partially or fully ionized gas instead of metal as the conducting medium to create an antenna. The advantages of plasma antennas are that they are highly reconfigurable and can be turned on and off. The radiated wave profile can be calculated by several numerical methods. The disadvantage is that plasma antennas require energy to be ionized. Hence, research to reduce the power required to ionize the gas at various plasma densities is important.
\end{abstract}

Keywords: Plasma antenna, Reconfigurable.

\section{Introduction}

The plasma state is a characterization of matter where long range electromagnetic interactions dominate the short range inter-atomic or intermolecular forces among a large number of particles. Since 1993 contributions to plasma antennas have mainly been made by a few groups in the United States and Australia. The Naval Research Laboratory in the United States developed the reflector plasma antenna (Manheimer, 1993; Mathew et al., 1995) called the Agile Mirror which could be oriented electronically and had the capability of providing electronic steering of a microwave beam in a radar or electronic warfare system.

Moisan et al. (1982) have proposed that a plasma column could be driven directly from one end by excitation of an RF plasma surface wave. Their work was the foundation research on plasma antennas in Australia. Borg et al., (1999; 2000) used surface waves to excite the plasma column and used one electrode to simplify the antenna design. The need for two electrodes is eliminated and the plasma column projects from the feed point. The frequency range studied was between $30 \mathrm{MHz}$ to $300 \mathrm{MHz}$.

In the United States, Anderson and Alexeff (2006; 2007 ; 2008) did theoretical work, experimented, and built prototypes of plasma antennas, plasma waveguides, and plasma frequency selective surfaces (FSS). Their research and development focused on theory and experiments to show advantages of plasma antennas, reducing the power required to ionize a plasma tube with higher plasma densities and frequencies, and the development of the smart plasma antenna. They have built and tested plasma antennas from $30 \mathrm{MHz}$ to 20 $\mathrm{GHz}$. They also have reduced the power required to maintain ionization in a plasma tube to an average power of 5 Watts or less at $20 \mathrm{GHz}$. This is much less than the power required turning on a fluorescent lamp. It is anticipated that power requirements will continue to decrease. Jenn (2003) wrote an excellent survey of plasma antennas, but much progress has been made since then. Plasma is mainly produced by the surface waves or enhanced microwave fields due to the resonant absorption arisen just under the dielectric window.

Fundamental plasma antenna theory

In the case of plasma antennas, plasma fluid models (Krall \& Trivelpiece, 1973; Chen, 1984; Balanis, 1997; Jenn, 2003;) can be used to calculated plasma antenna characteristics. For example, we proceed with the derivation of net radiated power from a center-fed dipole plasma antenna with triangular current to obtain an analytical solution. For simplicity, the equations are linearized and one dimension is considered with the plasma antenna dipole antenna oriented along the $z$-axis. The dielectric constant for the plasma is defined as

$\varepsilon=1-\frac{\omega_{p}^{2}}{\omega(\omega-j v)}$

where,

$\omega_{p}=\sqrt{\frac{n e^{2}}{\varepsilon_{0} m}}$

is the plasma frequency and $m$ is the mass of the electron, $v$ is the electron velocity in the fluid model, $e$ is the charge on the electron, $E$ is the electric field, $\omega$ is the applied frequency in radians per second. Assuming the plasma antenna is a center-fed dipole antenna with a triangular current distribution as given by Balanis (1997), we obtain for the dipole moment of the plasma antenna:

$\mathrm{P}=\mathrm{a} \frac{e^{2} n_{0} E_{0} d}{2 m\left[\omega(\omega+j v)-\omega_{p}^{2}\right]}$

where $a$ is the cross-sectional area of the plasma antenna and $d$ is the length of the plasma antenna. The total radiated power is then given by

$p_{\text {rad }}=\frac{k^{2} \omega^{2}}{12 \pi \varepsilon_{0} c}|p|^{2}$

where $k$ is the wave number. Substituting equation (3) into equation (4) yields

$p_{\text {rad }}=\left(\frac{\varepsilon_{0} a^{2}}{48 \pi c}\right)(k d)^{2}\left(\omega_{p}^{4}\right) \frac{\left(\omega E_{0}\right)^{2}}{\left[\left(\omega^{2}-\omega_{p}^{2}\right)^{2}+\nu^{2} \omega^{2}\right]}$

M.Pourbagher et al. Indian J.Sci.Technol.
Popular article

CIndian Society for Education and Environment (iSee)
"Reconfigurable plasma antenna" http://www.indjst.org 
In this last expression we see that the net radiated power for a plasma antenna is a function of the plasma frequency and collision rates.

\section{Plasma antenna windowing (Fundamental of the smart} plasma antenna)

Plasma antenna windowing is a term coined to describe an RF signal being transmitted through plasma tubes, which are off or low enough in plasma density that RF signals pass through. Various designs of plasma windows appear in patents by T. Anderson (U.S. Patent number 5,963,169; 7, 342, 549; 6, 922, 173; 6,870,517; Anderson, \& Alexeff, Appl. SI. No: 11/879, 725; Anderson, \& Alexeff, 2002). Papers and conference presentations on plasma windowing have been made by Anderson (2002). A detailed numerical analysis of the performance of a reconfigurable antenna comprised of a linear omni directional antenna surrounded by a cylindrical shell of conducting plasma is presented. The plasma shield consists of a series of tubes containing a gas (in practice, fluorescent light bulbs are used). The plasma is highly conducting and acts as a reflector for radiation for frequencies below the plasma frequency. Thus, when all of the tubes surrounding the antenna are electrified, the radiation is trapped inside.

By leaving one or more of the tubes in a non electrified state, apertures are formed in the plasma shield, which allow radiation to escape. This is the essence of the plasma window-based reconfigurable antenna. The apertures can be closed or opened rapidly (on microsecond time scales) simply by applying voltages.

Theoretical analysis and boundary value problem

The goal of the theoretical analysis is the prediction of the far-field radiation pattern of the plasma window antenna (PWA) for a given configuration. In order to simplify the analysis, we make the approximation that the length of the antenna and surrounding plasma tubes is irrelevant to the analysis, physically Assume a wire (the antenna) is located at the origin and carries a sinusoidal current of some specified frequency and amplitude. Next assume that the wire is surrounded by a collection of

Fig. 1. Geometry of 7 touching cylinders (one removed) with source and observation point

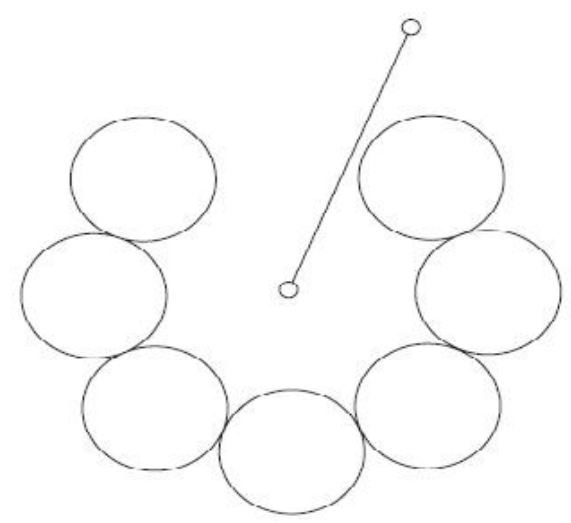

Popular article

CIndian Society for Education and Environment (iSee) cylindrical conductors each of the same radius and distance from the origin. Solve for the field distribution everywhere in space and thus obtain the radiation pattern.

One particular configuration for the PWA is illustrated in Fig. 1 for the case of seven cylinders. For a complete shield, assume $N$ cylinders are placed with their centers lying along a common circle chosen to have the source antenna as its center. Choose some distance from the origin $d$ and divide the circle of radius $d$ into equal segments subtending the angles

$\psi_{l}=2 \pi l / N$

where the integer / takes on the values $/=0,1, \ldots(N-1)$. The solution to the boundary value problem is obtained by assuming the cylinders to be perfect conductors, which forces the electric fields to have zero tangential components on the surfaces of the cylinders. Enforcing this condition on each of the cylinders leads to $N$ linear equations for the scattering coefficients. The consequence is an $N \times N$ linear algebraic problem, which is then solved by matrix inversion.

The field produced by a wire aligned with the $\hat{z}$-axis, which carries a current /is

$\vec{E}_{\text {inc }}^{*}(\rho)=-\left(\frac{I \pi k \hat{z}}{c}\right) H_{0}^{(1)}(\mathrm{k} \rho)$

Where $k$ is the wave vector defined by $k=\omega / c, c$ is the speed of light, and the angular frequency $\omega$ is given in terms of the $f$ by $\omega=2 \pi f$. The Hankle function of the first kind, of order $n$ (in this case $n=0$ ) is defined by

$H_{n}^{(1)}(\mathrm{x})=I_{n}(x)+\mathrm{i} Y_{n}(x)$

Where $I_{n}(x)$, and $Y_{n}(x)$ are the Bessel functions of the first and second kind, respectively. This assumes that all quantities have the sinusoidal time dependence given by the complex exponential with negative imaginary unit exp $(-i \omega t)$. The key to solving the present problem hinges on the fact that waves emanating from a given point (i.e., from the source or scattered from one of the cylinders) can be expressed as an infinite series of partial waves:

$\mathrm{E}(\rho, \Phi)=\hat{z} \sum_{m=-\infty}^{\times} A_{m} H_{m}(\mathrm{k} \rho) \exp (-\mathrm{im} \Phi)$

Where, the superscript on the Hankel function is dropped because any given term in the series can be expanded in a similar series in any other coordinate system by using the addition theorem for Hankel functions. The addition theorem for Hankel functions is written

$\operatorname{Exp}(\operatorname{in} \psi) H_{n}(k R)=\sum_{m=-\infty}^{x} J_{m}\left(k r^{\prime}\right) H_{n+m}(k r) \exp (\operatorname{im} \Phi)$ (10)

Where the three lengths $r^{\prime}, r$, and $R$, are three sides of a triangle such that 
Fig. 2. Plot of the radiated flux in the far field. This quantity is obtained by integrating the Poynting vector over a cylindrical surface of unit height in the far field. Physically, this quantity should not exceed unity. Situations in which values greater than unity are obtained indicate the presence of values, which lead to singular matrices.

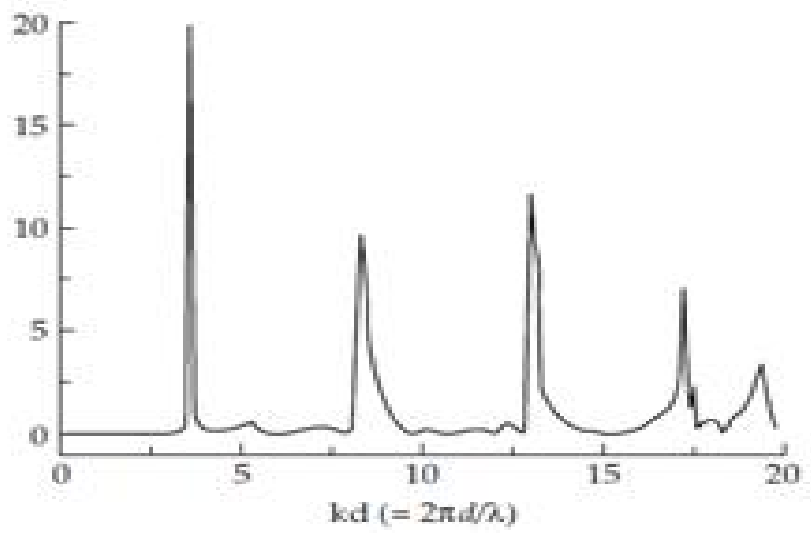

Fig. 3. Far-field radiation patterns for various solutions illustrated in Fig. 2

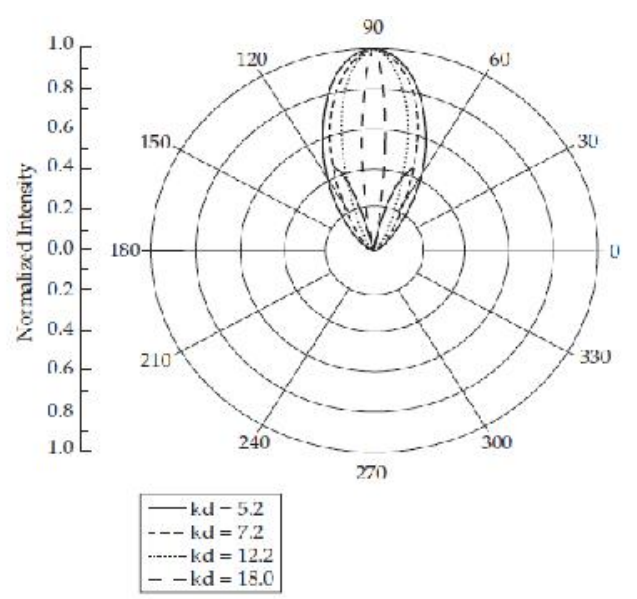

Fig. 4. Geometry of 15 touching cylinders (one removed) with source and observation point

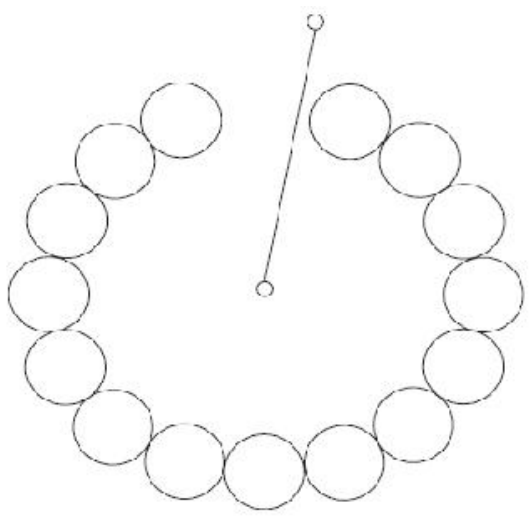

Vol. 5 No. 6 (June 2012)

ISSN: 0974- 6846
Fig.5. Plot of the far-field flux radiated from the 15cylinder plasma window antenna. As in Fig. 2, this plot includes all solutions including nonphysical solutions for which the flux exceeds unity.

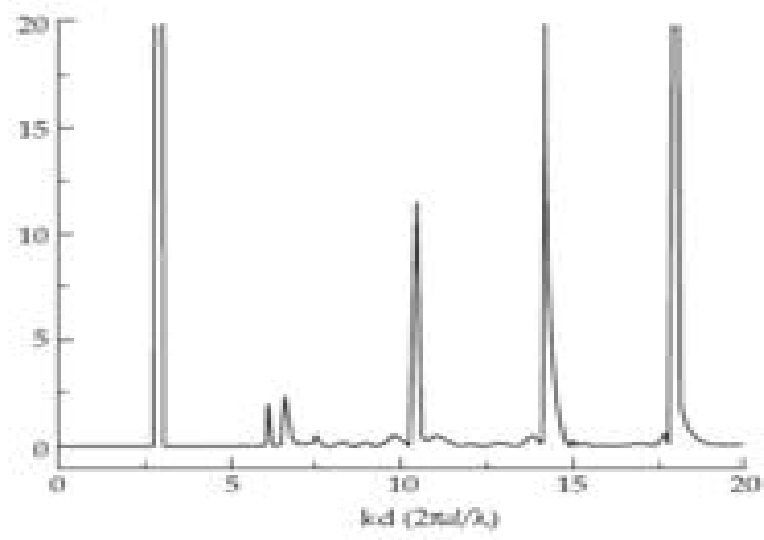

Fig. 6. Far-field radiation patters for the 15 cylinder plasma antenna for several of the solutions illustrated in Fig. 5

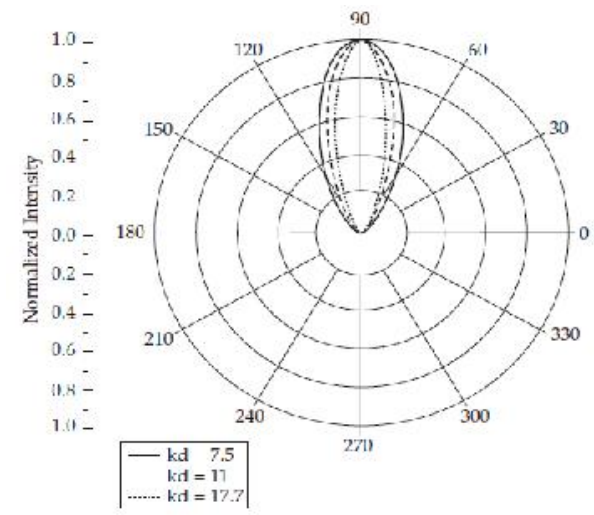

Fig. 7. Half beam-width versus wavelength for the two plasma window antenna configurations. This is defined as the angle at which the far-field radiation pattern is reduced by a factor of one half.

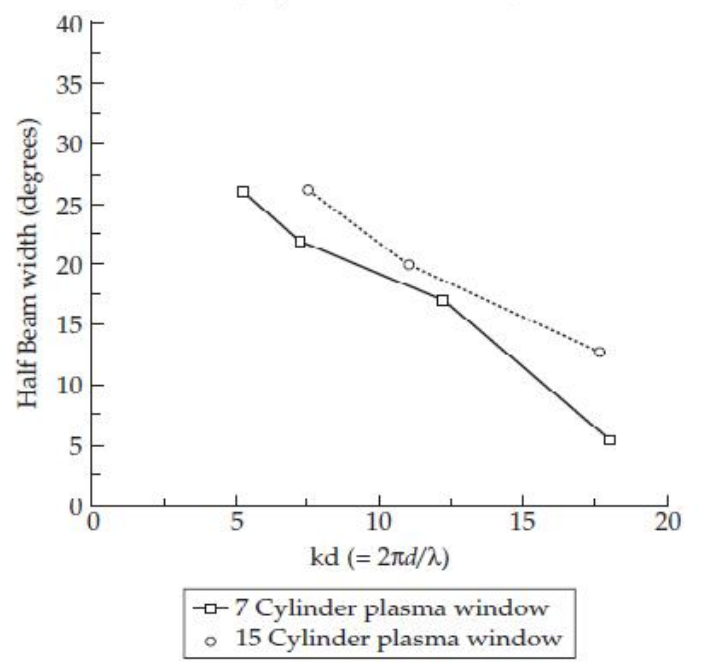

Popular article COIndian Society for Education and Environment (iSee)
"Reconfigurable plasma antenna" http://www.indjst.org
M.Pourbagher et al. Indian J.Sci.Technol. 
$\mathrm{R}=\sqrt{r^{\prime 2}+r^{2}-2 r r^{\prime} \cos (\Phi)}$

with $r^{\prime}<r$, and $\psi$ is the angle opposite to the side $r^{\prime}$. Another way to express this is as follows:

$\operatorname{Exp}(2 \mathrm{i} \psi)=\frac{r-r^{\prime} \exp (-i \Phi)}{r-r^{\prime} \exp (i \Phi)}$

\section{Far-Field Radiation Pattern}

Now, for convenience choose the amplitude of the source current so as to obtain unit flux in the absence of the cylinders. In other words, choose the source field to bere: given by

$\overrightarrow{\text { Eing: }}=-\sqrt{\frac{2 k \pi}{c}} H_{0}(\mathrm{k} \rho)$

Verifying that this gives unit flux. The far-field limit of the Hankel function is

$H_{m}(\mathrm{k} \rho) \simeq \sqrt{\frac{2}{\pi k \rho}} \exp \left[\mathrm{i}(\mathrm{k} \rho-(2 \mathrm{~m}+1))^{\pi} / 4\right]$

amids the magnemitic field is obtained from the electric field axs:

$\overrightarrow{B_{i}}=\frac{-i c}{\omega} \nabla \times \overrightarrow{E_{i m a x}}$

The radiation intensity is obtained from this field by computing the Poynting vector:

$\vec{p}=\frac{c}{8 \pi} \operatorname{Re}\left[E \times B^{*}\right]$

By integrating this over a cylindrical surface of unit height, at a distance $r$, the unit flux is obtained. By extracting a factor of $\sqrt{\frac{2 k \pi}{c}}$, the total electric field can be expressed as

$\vec{E}:=-\sqrt{\frac{2 k \pi}{c}}\left(H_{0}(k \rho)-\sum_{n=-M}^{M} B_{n} H_{n}(k \rho) \exp (\operatorname{in} \Phi)\right)$

The far-field radiation pattern is obtained by plotting the radial component of the Poynting vector at a given distance (in the far field) as a function of angle. Plots of radiated power and radiation patterns of the antenna configuration of Fig. 1 are given in Fig. 2 and 3. The next geometry of the PWA is given in Fig. 4 with 15 touching cylinders and one removed is given in Fig. 4. The radiated power and radiation pattern of the PWA given in Fig. 4 is given in Figs. 5 and 6. Fig. 7 shows the half beam width vs wavelength for the PWA configurations of Fig. 1 and Fig. 3.
Vol. 5 No. 6 (June 2012)

ISSN: 0974- 6846
Latest developments on plasma antennas and fabry-perot resonator

One of the remarkable plasma effects experimentally discovered is the large increase in plasma density at the same average power input provided by pulsing the power input (Hojo, 2004; Shono, 2008). In our experiments, a density increase of over 100 has been observed. Although various experimenters have observed similar effects using different power input techniques, to our knowledge no one has yet provided a theoretical explanation.

The characteristic decay time of the plasma after power turn-off is typically many milliseconds, so the opening time of such a barrier generally is also predicted also to be many milliseconds. However, the plasma barrier can be opened and closed in faster time scales. This can be done by increasing the plasma density rather than waiting for it to decay.

If a standing wave is produced between the two layers of plasma that results in microwave transmission, this may be analogous to the transmission found in an optical Fabry-Perot Resonator. The secret lies in the boundary layer behavior of the plasma. Once microwave cutoff occurs, one would expect the plasma behavior to be static. What actually occurs is that at microwave cutoff, the reflection is in-phase with the incident wave, in analogy to an open coaxial line (The electron and displacement currents are equal, but out of phase). As the plasma density further increases, the reflection smoothly changes from in phase to $180^{\circ}$ out of phase, in analogy to a shorted coaxial line (The reflection current is much greater than the displacement current). The boundary condition at a vacuum plasma interface of the reflected electric field in terms of the incident electric field is

$E_{r}=\left(\frac{1-i \beta}{1+i \beta}\right) E_{0}$

Where the phase shift is given by

$\beta=\sqrt{\frac{\omega_{p}^{2}}{\omega^{2}}-1}$

The consequence of this phase shift is that, given any kind of a plasma resonator, if the plasma density is raised high enough, the resonance required for the Fabry-Perot effect to take place must occur.

\section{Conclusion}

This paper reviews recent work and applications of plasma antennas. The advantages of plasma antennas are that they are highly reconfigurable and can be turned on and off. The disadvantage is that plasma antennas require energy to be ionized. Hence, research to reduce the power required to ionize the gas at various plasma densities is important.
"Reconfigurable plasma antenna" http://www.indjst.org
M.Pourbagher et al. Indian J.Sci.Technol. 


\section{References}

1. Alexeff and Anderson $T$ (2008) Recent results of plasma antennas. Phys. Plasmas. 15, 057104.

2. Alexeff I. Pulsed plasma element. U.S. patent. 7, 274333.

3. Alexeff $I$ and Anderson T (2006) Experimental and theoretical results with plasma antennas. IEEE Trans. Plasma Sci. 34(2). doi:10.1109/TPS.2006.872180.

4. Anderson $T$ (2002) Storage and release of electromagnetic waves by plasma antennas and waveguides. pp. 74-82, 33rd AlAA Plasma dynamics \& Lasers Conf.

5. Anderson T. Configurable arrays for steerable antennas and wireless network incorporating the steerable antennas. U.S. patent 6, 870-517.

6. Anderson T. Multiple tube plasma antenna U.S. Patent number. 5, 963-169.

7. Anderson T. Reconfigurable scanner and RFID system using the scanner. U.S. patent $6,922,173$.

8. Anderson T. Tunable plasma frequency devices. U.S. patent. 7, 403-453.

9. Anderson T. Configurable arrays for steerable antennas and wireless network incorporating the steerable antennas. U.S. patent 7, 342-549.

10.Anderson $T$ and Alexeff I (2002) Theory and experiments of plasma antenna radiation emitted through plasma apertures or windows with suppressed back and side lobes. Intl. Conf. Plasma Sci. 2002.

11.Anderson $T$ and Alexeff I (2007) Plasma frequency selective surfaces. IEEE Trans. Plasma Sci. 35(2), 407.

12.Anderson $\mathrm{T}$ and Alexeff I. Reconfigurable scanner and RFID. Application Serial Number 11/879, 725.

13.Balanis C (1997) Antenna Theory, 2nd ed., pp: 143.

14.Borg G, Harris J, Martin N, Thorncraft D, Milliken R, Miljak D, Kwan D, Ng T and Kircher J (2000) Plasmas as antennas: Theory, experiment, and applications. Phys. Plasmas. 7(5), 2198, May 2000.

15. Borg GG, Miljak DG, Harris JH and Martin NM (1999) Appl. Phys. Lett. 74, pp: 3272.

16. Chen $F$ (1984) Introduction to plasma physics and controlled fusion. 2nd ed., Plenum Press.1, pp: 53-78.

17. Hojo H, Shimamura, A, Uchida N, Yasaka Y and Mase A (2004) Surface wave analysis with plasma resonance. J. Plasma Fusion Res. 80, 719.

18.Jenn DC (2003) Plasma antennas: Survey of techniques and the current state of the Art. Naval Postgraduate School. pp: 29.

19. Krall N and Trivelpiece A (1973) Principals of plasma physics. McGraw-Hill Inc. pp: 84-98.

20. Manheimer W (2008) Plasma reflectors for electronic beam steering in radar systems. IEEE Trans. Actions on Plasma Sci. 19(6), 1228.

21. Mathew J, Meger R, Gregor J, Pechacek R, Fernsler R, Manheimer W and Robson A (1995) Electronically steerable plasma mirror for radar applications. IEEE Intl. Radar Conf. pp.742.
Vol. 5 No. 6 (June 2012)

ISSN: 0974- 6846

22. Moisan M, Shivarova A and Trivelpiece AW (1982) Phys. Plasmas. 20, pp: 1331

23. Shono H, Yasaka Y, Tsuji A and Takeno H (2008) Present at ICPP2008. Intl. Congress on Plasma Phys.
"Reconfigurable plasma antenna" http://www.indjst.org
M.Pourbagher et al. Indian J.Sci.Technol. 